\title{
Cambio climático global: una realidad inequívoca
}

\author{
Global Climate Change: An Unequivocal Reality
}

\author{
Raynal-Villaseñor J.A. \\ Departamento de Ingeniería Civil y Ambiental \\ Escuela de Ingeniería \\ Universidad de las Américas, Puebla \\ E-mail: josea.raynal@udlap.mx
}

Información del artículo: recibido: agosto de 2009, reevaluado: julio de 2010, aceptado: noviembre de 2010

\section{Resumen}

Durante varios años se ha discutido si existe un cambio climático global y, si lo hay, cuál es su causa. Una vez publicado el 4o. Reporte de Valoración del Panel Intergubernamental sobre Cambio Climático (IPCC, 2007) -el IPCC es parte de la Organización de las Naciones Unidas (ONU)- se precisó que hay un cambio climático global en desarrollo y la causa inequívoca que lo está produciendo es la actividad humana en el planeta Tierra, también se habló en el IPCC de las causas naturales por las cuales el planeta se está calentando. En el presente artículo, se da información relevante al cambio climático global en desarrollo y se mencionan algunas acciones que cada ser humano de este planeta puede implementar para mitigarlo, ya que es imposible detenerlo.

\section{Abstract}

During several years, a long discussion has taken place over the reality of global climate change phenomenon and, if there is one, what could be its cause. Once the 4th Assessment Report of the Intergovernmental Panel on Climatic Change (IPCC, 2007) -IPCC is part the United Nations Organization (UN)- was published, it was stated that there is a developing global climatic change and that the cause is unequivocally related with the human activity in the planet Earth. In this paper, relevant information is given about the development of global climatic change issues and some actions are mentioned that each human being of this planet can implement to mitigate it, since it has been accepted that it's impossible to stop it.

\section{Descriptores}

- cambio climático global

- efecto invernadero

- gases de efecto invernadero

- medidas de mitigación para el cambio climático global

- dióxido de carbono

- metano

- óxido nitroso

\section{Keywords}

- global climate change

- greenhouse effect

- greenhouse effect gases

- global climate change mitigation measures

- carbon dioxide

- metane

- nitrous oxide 


\section{La controversia sobre el cambio climático ya terminó}

Durante varios años se ha especulado si realmente existe un cambio climático global a corto plazo en el planeta Tierra que, entre otras cosas, provocará una elevación significativa en la temperatura ambiental del mundo, encargada de desencadenar una serie de efectos en la elevación del nivel medio del mar, el incremento en la evaporación en suelos y superficies de agua, cambio en los patrones de lluvia, alteración de los ecosistemas, etcétera (Dimento et al., 2007). Esta controversia se terminó al publicarse el $4^{\mathrm{o}}$ Reporte del Panel Intergubernamental sobre Cambio Climático (IPCC) en 2007, en donde se afirmó categóricamente que las actividades humanas son las responsables del incremento en el contenido atmosférico de los gases de efecto invernadero (GEI) y, por consiguiente, del cambio climático global que está en desarrollo. Recientemente, apareció otro reporte llamado "Global Climate Change Impacts in the United States" (Showstack, 2009), que apoya lo expuesto en el $4^{\circ}$ Reporte del Panel Intergubernamental sobre Cambio Climático (IPCC, 2007).

\section{El efecto invernadero}

El efecto invernadero toma su nombre al compararlo con el efecto del proceso térmico, el cual se produce precisamente en un invernadero (figura 1), que es una construcción con paredes y techo de vidrio o de cualquier otro material transparente o translúcido, el cual está diseñado para atrapar parte de la radiación de onda larga y producir una elevación en la temperatura interior con respecto a la temperatura exterior del invernadero. Esta estructura que provoca un diferencial térmico con respecto al exterior, se utiliza para cultivar diversos productos vegetales, por ejemplo, permite cultivar y cosechar en pleno invierno fresas en Alemania.
El efecto invernadero se puede entender con un ejemplo cotidiano. Se puede percibir el efecto invernadero al subirnos a un auto, digamos entre 2:00 y 3:00 PM, después de dejarlo expuesto a los rayos del Sol toda la mañana. Al subir al auto, se percibe que está más caliente en su interior con respecto al espacio exterior que lo rodea, éste es el resultado de un incremento térmico que produce el efecto invernadero.

A escala atmosférica, el efecto invernadero ha existido en la Tierra desde la aparición en el planeta del agua en forma líquida, el principal GEI (Gas de Efecto Invernadero) es el vapor de agua (figura 2). Este compuesto (conjuntamente con otros gases como el oxígeno $\left(\mathrm{O}_{2}\right)$ y el dióxido de carbono $\left(\mathrm{CO}_{2}\right)$, ha producido un ambiente propicio para la aparición y evolución de la vida, sin él la temperatura promedio de la Tierra sería de $-18^{\circ} \mathrm{C}$ y el planeta sería una esfera con cobertura de hielo; en lugar de este escenario, la superficie terrestre tiene una temperatura promedio de $14^{\circ} \mathrm{C}$.

El vapor de agua, el $\mathrm{CO}_{2}$ y otros gases cuyas moléculas tienen momento eléctrico bipolar, absorben mayor radiación en la parte de longitud de onda larga del espectro electromagnético ocupado por la radiación terrestre saliente (onda larga), que en la parte de onda corta ocupada por la radiación solar incidente. Entonces un porcentaje de la radiación solar es reflejada por la atmósfera y la superficie terrestre, otro porcentaje pasa a través de la atmósfera libre de obstáculos y es absorbido por la superficie terrestre y reemitido a la atmósfera.

Parte de esta radiación infrarroja es absorbida y reemitida por las moléculas del gas de invernadero, calentando la superficie terrestre y la troposfera.

En el efecto invernadero a escala atmosférica, los gases de invernadero (vapor de agua, dióxido de carbono $\left(\mathrm{CO}_{2}\right)$, metano $\left(\mathrm{CH}_{4}\right)$, óxido nitroso $(\mathrm{NO})$ y los clorofluoro-carbonos (CFCs), actúan como los cristales en un invernadero.

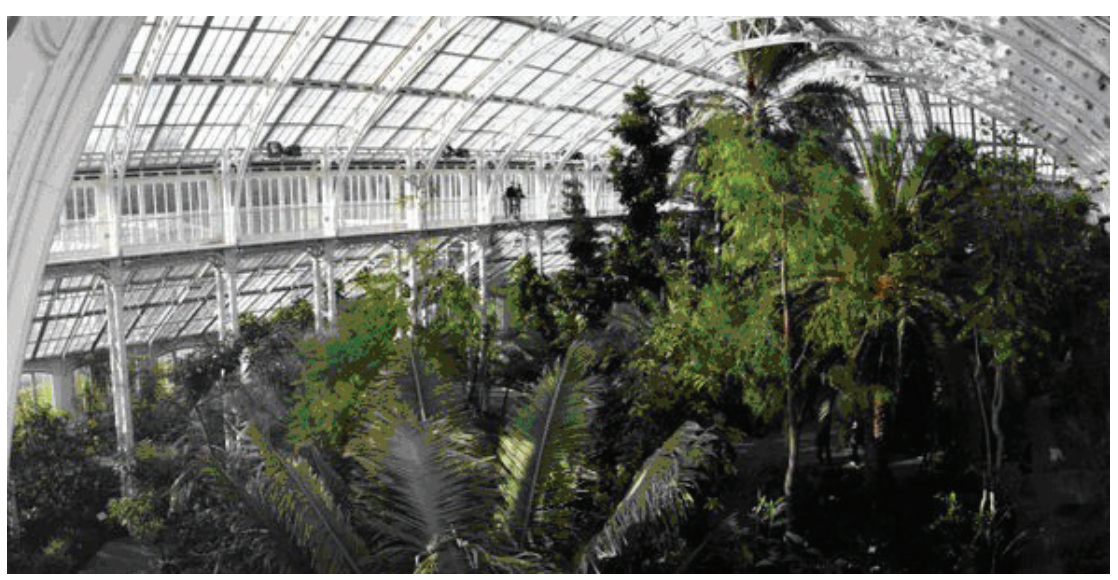

Figura 1. Invernadero 


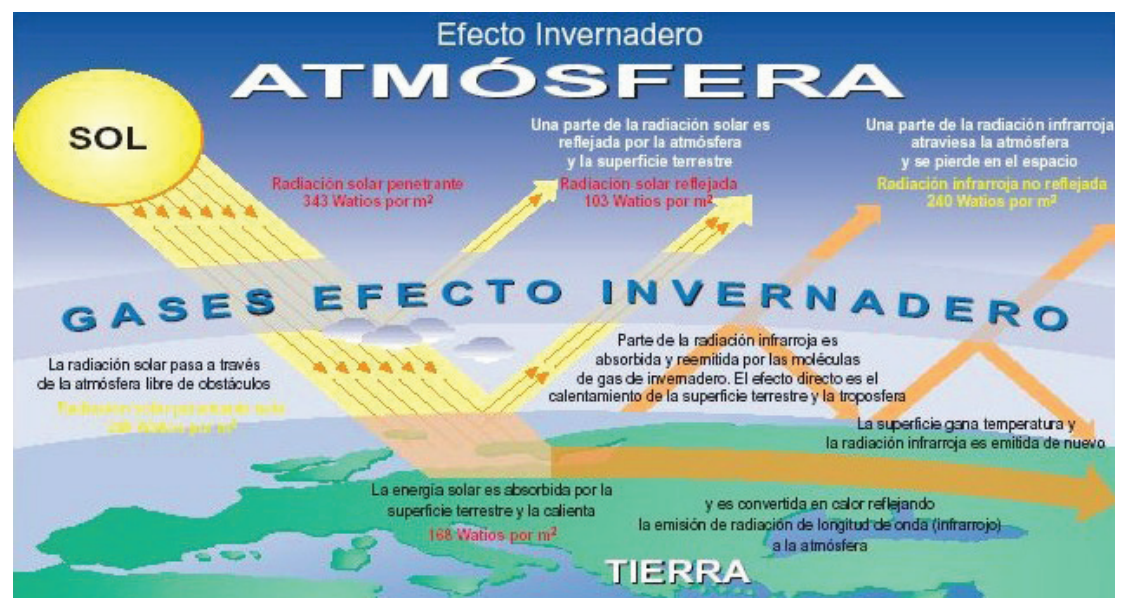

Figura 2. Esquema del efecto invernadero a escala atmosférica (UNEP-GRID-Arendal, 2007)
Los niveles de $\mathrm{CO}_{2}$, principal causante del efecto invernadero global actual, han estado aumentando en forma alarmante desde la revolución industrial, iniciándose un ascenso vertiginoso desde finales de los años cincuenta hasta la fecha (figura 3). Desde la Revolución Industrial hasta 1999, la concentración de $\mathrm{CO}_{2}$ en la atmósfera se ha incrementado $40 \%$, alcanzando un nivel de concentración que no ha sido excedido en los últimos 650,000 años y muy probablemente en los últimos 20 millones de años (Van Lenten, 2005). Se estima que las emisiones de $\mathrm{CO}_{2}$, observadas en el año 2002, serán rebasadas en 63\% para el año 2030 (RSC, 2005).

En la figura 4 se muestran las principales actividades humanas productoras de los GEI.

Recientemente, se mencionó que un nivel adecuado de la concentración del $\mathrm{CO}_{2}$ en la atmósfera debía mantenerse por debajo de las 400 partes por millón para evitar una elevación de la temperatura ambiental superior a $2^{\circ} \mathrm{C}$ (RSC, 2005). A finales de 2002, el nivel de concentración de $\mathrm{CO}_{2}$ en la atmósfera era del orden de 378

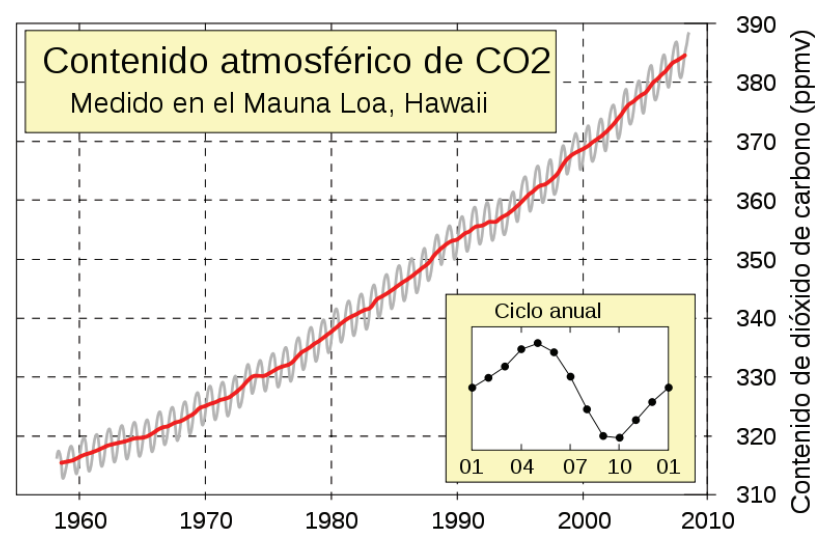

Figura 3. Variación mensual y anual en la concentración de $\mathrm{CO}_{2}$ (1959-2007).

(Valores obtenidos en Mauna Loa, Hawai, EUA) (IPCC, 2007) ppm y se estima que está subiendo a un ritmo de 2 ppm por año. Esto significa que, de seguir al mismo ritmo, el nivel de 400 ppm de concentración de $\mathrm{CO}_{2}$ se alcanzará en la atmósfera a finales del año 2013.

En abril de 2010 se reportó un nivel de concentración de 391 ppm de $\mathrm{CO}_{2}$ en la atmósfera (Wikipedia, 2010).

\section{El ascenso de la temperatura del aire}

El principal efecto disparador del cambio climático es la elevación de la temperatura del aire. El año 2009 fue el segundo más cálido, anteriormente lo había sido el 2007, a pesar de haber caído en un periodo de mínima radiación solar y durante el efecto de La Niña, que tendió a bajar la temperatura del planeta por medio de la disminución de la temperatura del Océano Pacífico. El 2010 se perfiló como uno de los más cálidos también. En los registros que se han tomado desde 1880 a la fecha, el año de 1998 estuvo en la primera posición del registro

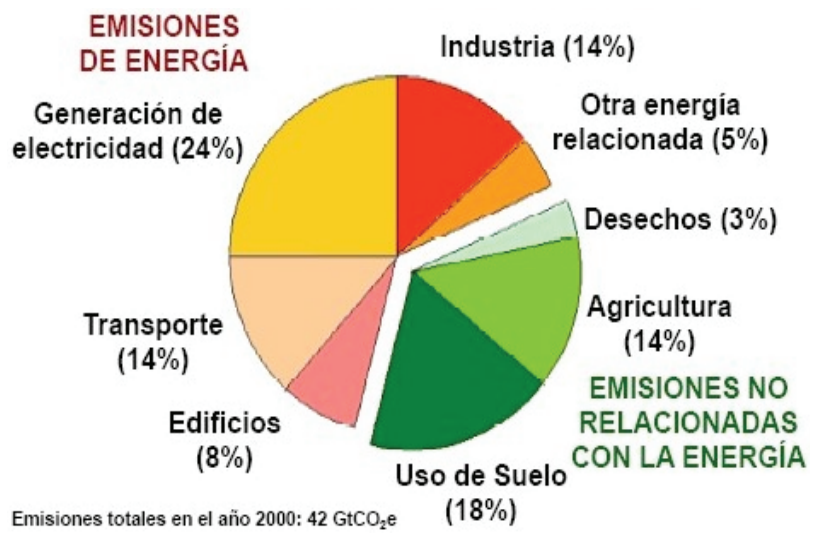

Figura 4. Actividades humanas que contribuyen a las emisiones de Gases de Efecto Invernadero (GEI) para el año 2000 en giga toneladas de $\mathrm{CO}_{2}$ equivalentes (Wikipedia, 2010) 
por ser el año más cálido, que coincidió con la aparición del fenómeno conocido como El Niño, el cual se caracterizó por un aumento de la temperatura en el Océano Pacífico, el cual contribuyó a elevar la temperatura del planeta (IPCC, 2007). La década de 2000-2009 fue la más cálida registrada desde 1880 (ADA, 2010). En el año 2001, se predijo que la elevación de la temperatura ambiental durante el siglo XXI alcanzaría incrementos desde $1.4^{\circ} \mathrm{C}$ hasta $5.8^{\circ} \mathrm{C}$ (IPCC, 2001). Estos incrementos en la temperatura ambiental se agudizarían en el Polo Norte, esperando en ese lugar un incremento adicional del orden de $50 \%$ más. Por ello, se recomienda que el incremento en la temperatura ambiental se mantenga por debajo de $2.5^{\circ} \mathrm{C}$ en el planeta, $4^{\circ} \mathrm{C}$ en el Polo Norte y que el nivel medio del mar no suba más de 1 metro (RSC, 2005).

\section{El nivel del mar a través de los tiempos}

Uno de los efectos más temidos del cambio climático es el ascenso del nivel medio del mar, ya que al subir unos cuantos metros, quedarían bajo el agua todas las ciudades costeras del mundo; también habría muchas islas que desaparecerían del mapa terráqueo inundadas por el océano.

El nivel medio del mar se ha modificado en los últimos 600,000 años, $100 \mathrm{~m}$ por arriba y $100 \mathrm{~m}$ por abajo del nivel actual (figura 5). Al inicio del Plioceno, hace 12 millones de años, el nivel del mar estuvo $380 \mathrm{~m}$ por arriba del nivel actual (Sellers, 1965).

Se encontró que al final de la era glacial más reciente, el mar elevó su nivel abruptamente al generarse una ola de más de $100 \mathrm{~m}$ de altura, la cual provocó un cambio ascendente en el nivel del mar de alrededor de $140 \mathrm{~m}$. Esto provocó que más de 26 millones de $\mathrm{km}^{2}$ quedaran bajo el agua de océanos y mares. Se sabe que una elevación de $1^{\circ} \mathrm{C}$ en la temperatura del agua de mar provocaría el derretimiento del hielo perenne en el Polo Norte en un área de 1.5 millones de $\mathrm{km}^{2}$ (RSC, 2005).

Un aumento de $3^{\circ} \mathrm{C}$ en el Polo Norte, produciría, a largo plazo, el derretimiento total del hielo de Groenlandia. Se ha estimado que si se derritiera totalmente la capa de hielo que forma a Groenlandia, el mar subiría su nivel aproximadamente $7 \mathrm{~m}$ (IPCC, 2007). Hay que resaltar que el volumen de hielo contenido en Groenlandia es una parte importante del Polo Norte.

Por otro lado, se infiere que bajo las tendencias actuales, el derretimiento del Polo Norte implicaría una elevación del nivel medio del mar de $7 \mathrm{~m}$ por encima del nivel actual, la ocurrencia de este hecho se consideraba para los veranos a partir del año 2040. Actualmente, 2040 se considera muy optimista y se espera que entre el año 2013 y el año 2030 ocurra el primer derretimiento total del Polo Norte en los veranos (National Geographic, 2008). En la figura 6 se presenta un mapa del Golfo de México, del Mar Caribe y del Océano Atlántico occidental en donde se muestran las áreas continentales que quedarían sumergidas si se presentara una elevación en el nivel medio del mar de $6 \mathrm{~m}$, respecto al nivel medio del mar actual (Rowley et al, 2007). Como puede observarse, México perdería Cozumel, la isla más grande del territorio nacional, Cancún y la Riviera Maya, en donde se tiene la zona hotelera más importante del País, gran parte de las zonas costeras de la península de Yucatán y de los estados de Veracruz, Campeche, Tabasco y Tamaulipas.

Mundialmente, se estima que por cada metro de elevación en el nivel medio del mar, se tendrían millones de personas afectadas como lo muestra la tabla 1.

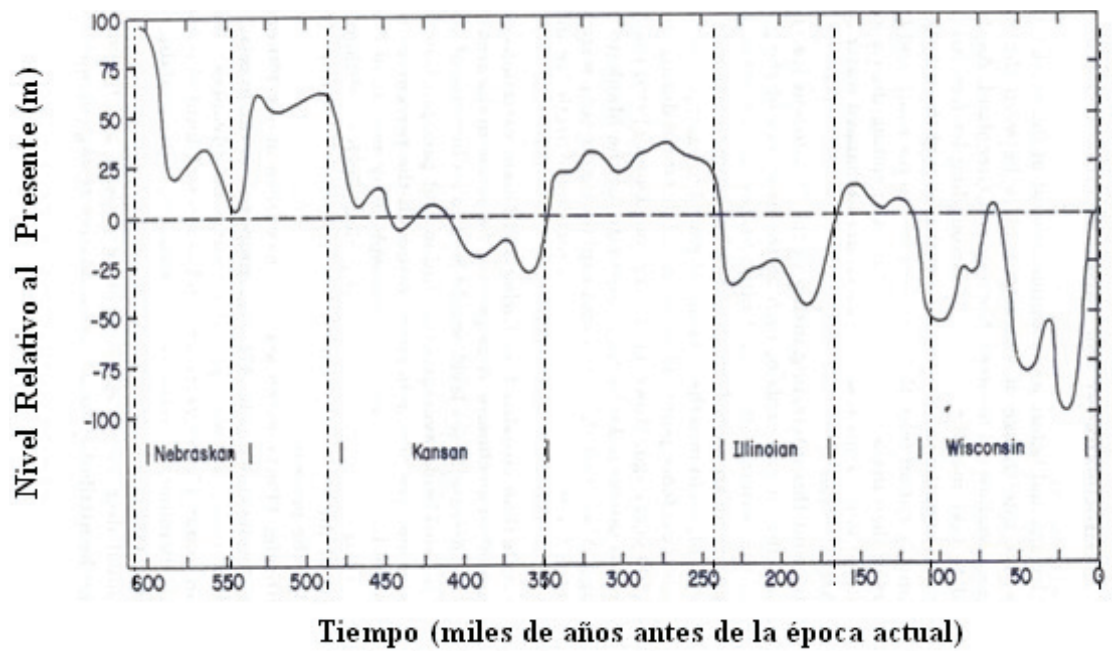

Figura 5. Variación del nivel medio del mar en los últimos 600,000 años (Sellers, 1966) 


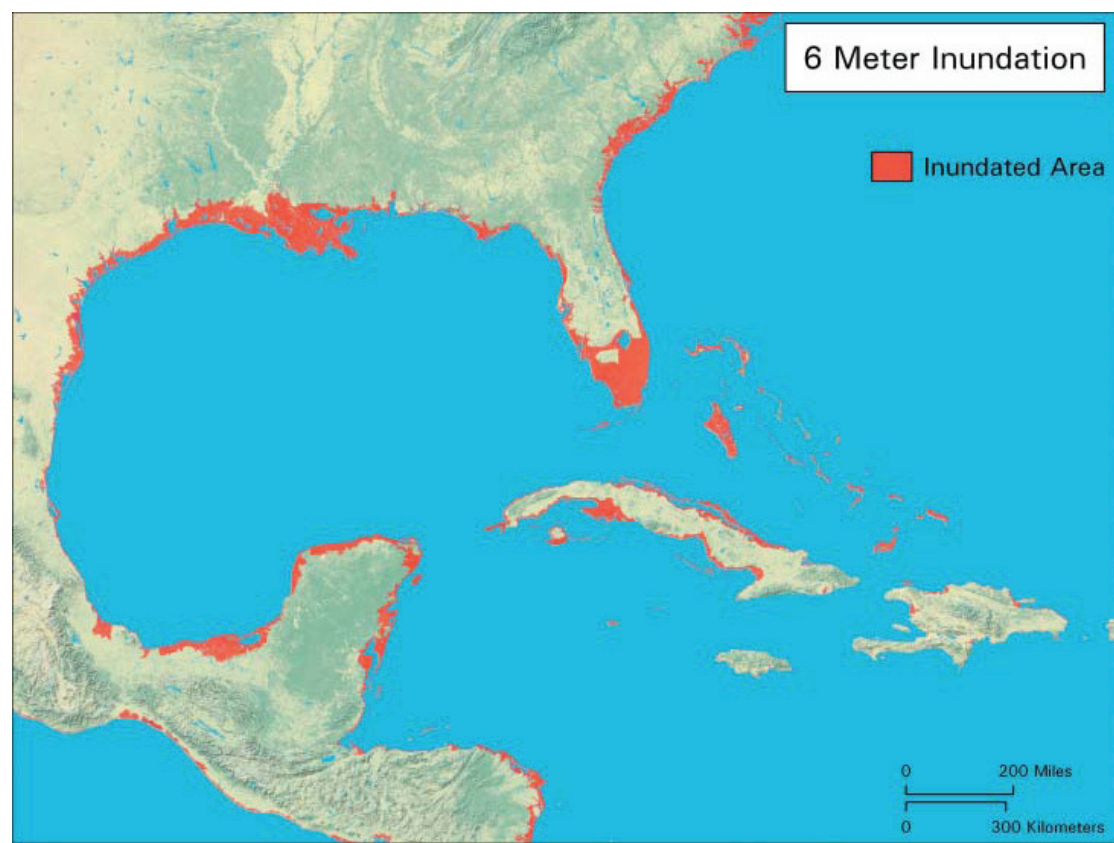

\begin{tabular}{ccc}
\hline $\begin{array}{c}\text { Incremento en el nivel medio del mar } \\
(\mathrm{m})\end{array}$ & $\begin{array}{c}\text { Área inundada } \\
\left.\text { (millones de } \mathrm{km}^{2}\right)\end{array}$ & $\begin{array}{c}\text { Población afectada } \\
(\text { millones de personas) }\end{array}$ \\
\hline 1 & 1.05 & 108 \\
2 & 1.31 & 175 \\
3 & 1.54 & 234 \\
4 & 1.78 & 308 \\
5 & 2.00 & 376 \\
6 & 2.19 & 431 \\
\hline
\end{tabular}

Figura 6. Áreas que se inundarán en las zonas costeras del Golfo de México, del Mar Caribe y del Océano Atlántico occidental al elevarse 6 metros el nivel medio del mar con respecto al nivel actual (Rowley et al., 2007)

Tabla 1. Población afectada y área inundada por cada metro de incremento en el nivel medio del mar (Rowley et al., 2007)

\section{El incremento en la evaporación potencial en suelo y agua}

Un factor del cambio climático global que no ha sido plenamente sopesado y mucho menos evaluado, es el relativo al incremento de la evaporación potencial del agua en el suelo y en los cuerpos de agua, como son océanos, mares, lagunas y lagos naturales y artificiales; estos últimos creados por presas de almacenamiento. El incremento en la evaporación potencial provocará la necesidad de una mayor cantidad de agua para producir productos agrícolas, tanto para consumo humano como para consumo animal e incidirá también en la generación de productos pecuarios.

Los efectos sobre los recursos forestales serán importantes, principalmente por estar sometidos a una mayor evapotranspiración y en algunas zonas a una baja importante en la precipitación. Otro aspecto significativo son las nuevas plagas a que estarán expuestos por la migración de éstas a latitudes más altas. Las plagas que existen ahora serán más resistentes al reducirse el número de días con heladas durante el año. El incremento de los incendios forestales es otro asunto que afectará severamente a los recursos forestales (IPCC, 2007).

La evaporación potencial en los océanos y mares producirá un mayor flujo de agua hacia la atmósfera, que interactuará de manera importante en la generación de ciclones tropicales, para los cuales se pronostica un aumento en número y en intensidad para los próximos años (Emanuel, 2005).

\section{El crecimiento poblacional y sus implicaciones en el cambio climático global}

La población de seres humanos en la Tierra ha crecido de forma acelerada después de la revolución industrial (figura 7; EUMED, 2010). La población mundial tardó varios millones de años en llegar al primer millón de seres humanos ocurrido hace 300,000 años. Hace 10,000 años ya existían 5 millones de seres humanos. $\mathrm{Al}$ inicio de la Era Cristiana, hace 2,000 años, la población era de 150 millones de personas. La población se incrementó a 500 millones de habitantes en el siglo XVI. Para el año 1900, el número de habitantes de la Tierra era 1,500 millones. En los años 60 el número anterior se duplicó y en 


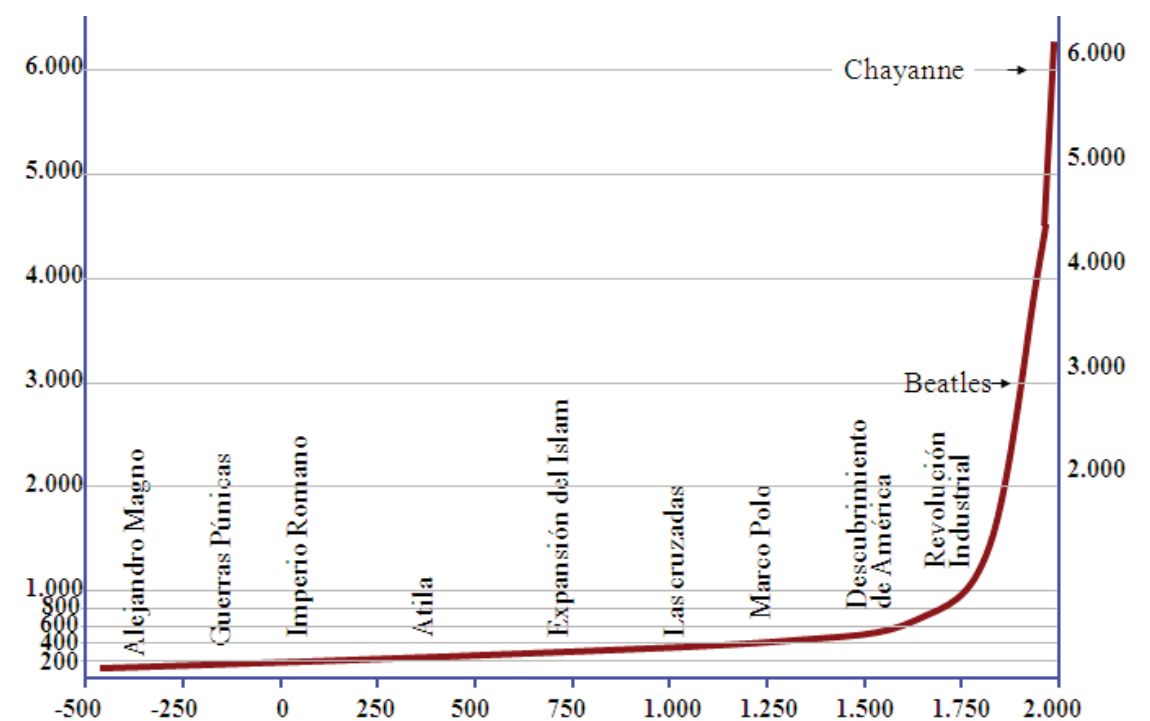

Figura 7. Crecimiento poblacional en los últimos 2500 años,(EUMED, 2010)
1999 llegó a 6,000 millones. Actualmente, la población del planeta se estima en 6,834 millones de seres humanos y se espera que llegue a 7,959 millones en 2050, según el pronóstico más optimista (Milenio, 2010).

La repercusión que tiene el crecimiento de la población en el calentamiento y cambio climático globales es muy importante. Ese crecimiento está ligado a una mayor producción de bienes y servicios que se basan en el consumo de combustibles y materiales provenientes del petróleo y del carbón, principalmente. La agricultura intensiva requerida para producir más alimentos, el transporte, el cambio en el uso del suelo, la generación de electricidad, la industria, etcétera, generan una gran cantidad de GEI, como efecto colateral, como puede observarse en la figura 4.

\section{¿Qué se puede hacer para mitigar los efectos del cambio climático global?}

Cada uno de nosotros debe contribuir a disminuir el crecimiento de los gases de invernadero en la atmósfera terrestre. El control del crecimiento de la población humana a nivel mundial es una prioridad que todos los gobiernos debieran atender. Es necesario bajar las emisiones de dióxido de carbono, metano y óxido nitroso a la atmósfera reduciendo el uso del automóvil, comprando automóviles híbridos, eléctricos o de aire comprimido para disminuir el consumo de gasolina, sustituir los aparatos domésticos por unos más eficientes, cambiar nuestros focos de filamento incandescente por los de luz fría y tomar muchas otras medidas que pueden consultarse en Internet o en video, siendo la mejor opción en forma global el documental sobre Al Gore, algunos ejemplos son:
1. An Inconvenient Truth, Al Gore, Paramount Studios, 2007. Disponible en centros de renta de videos o para compra en: www.amazon.com

2. www.climatechange.com

3. http://en.wikipedia.org/wiki/Climate_change_mitigation

4. http://www.oecd.org/dataoecd/30/41/41753450.pdf

5. http://portal.unesco.org/science/es/ev.php-URL_ ID $=7864 \& U R L \_D O=D O \_T O P I C \& U R L \_S E C-$ TION=201.html

6. http://www.unep.org/publications/ebooks/kickthe-habit/Default.aspx?bid=ID0EWHAC

Por otra parte, es necesario participar en campañas de reforestación, protección y recuperación de zonas forestales, ya que se estima que cada ser humano produce tal cantidad de $\mathrm{CO}_{2}$ a lo largo de su vida, que se requerirían 500 árboles para transformar esa cantidad en oxígeno.

En zonas urbanas, es importante concientizar a la gente de la importancia de mantener las áreas verdes, que permitan la permanencia de una cobertura vegetal y de zonas con infiltración natural y que permitan la recarga de acuíferos.

Un pequeño y permanente esfuerzo de todos los habitantes del planeta podrá más que el quehacer de cualquier gobierno. Existe aún la esperanza de que esta bomba de tiempo se pueda desactivar; pero debemos hacer uso de nuestras capacidades de supervivencia como raza para prevalecer al temporal que tendremos que enfrentar en los años por venir. 


\section{Agradecimientos}

El autor agradece a la Universidad de las Américas, Puebla el apoyo otorgado para la realización de este artículo.

\section{Referencias}

Análisis del Discurso Ambiental (ADA). La última década, la más cálida [en línea]. (Fecha de consulta 30 de julio de 2010) Disponible en: http://analisisdeldiscursoambiental.blogspot. com/ 2010/04/la-ultima-decada-la-mas-calida-desde.html

DiMento J.F.C. y Doughman P. Climate Change, The MIT Press, 2007.

Emanuel K. Increasing Destructiveness of Tropical Cyclones Over the Past 30 Years. Nature, 436(4), 2005

EUMED, Evolución de la población humana 2007 [en línea]. (Fecha de consulta 30 de Julio de 2010). Disponible en: www.eumed.net/cursecon/ppp/poblacion.ppt,

Intergovernmental Panel on Climate Change (IPCC), 3rd. Assessment Report, 2001 [en línea]. (Fecha de consulta 30 de Julio de 2010). Disponible en: http://translate.google.com.mx/tra nslate?hl=es\&langpair=en\%7Ces\&u=http://www.grida.no/climate/ipcc_tar/wg1/index.htm

Intergovernmental Panel on Climate Change (IPCC), 4rd. Assessment Report, 20072007 [en línea]. (Fecha de consulta 30 de Julio de 2010). Disponible en: http://www.wmo.int/pages/ partners/ipcc/index_en.html
Milenio. Día mundial de la población 2010: "Todos contamos", 2010. [en línea]. (Fecha de consulta 30 de Julio de 2010). Disponible en: http://www.milenio.com/node/483532

National Geographic, North Pole May Be Ice-Free for First Time This Summer, 2008 [en línea]. (Fecha de consulta 30 de Julio de 2010). Disponible en: http://news.nationalgeographic.com/ news/2008/06/080620-north-pole.html

Report of the Steering Committee (RSC), International Symposium on the Stabilisation of Greenhouse Gases. Exeter, Inglaterra, 16 p, 2005.

Rowley R.J., Kostelnick J.C., Braaten D., Li X. y Meisel J. Risk of Rising Sea Level to Population and Land Area. EOS, 88(9):105, 27, February, 2007

Sellers-William D. Physical Climatology, The University Chicago Press, Chicago, Illinois, EUA, pp. 197-228, 1965.

Showstack R. Climate Change Now Apparent and Unequivocal, New Report Warns. EOS, 90(26):223, 30 June, 2009.

UNEP-GRID-Arendal, Climate, 2007 [en línea]. (Fecha de consulta 30 de Julio de 2010). Disponible en: http://www.grida.no/ publications/vg/climate/page/3058.aspx

Van-Lenten C. The Slow-Motion Tsunami: How Climate Change Could Change the World, Update. The New York Academy of Sciences Magazine, pp. 2-7, marzo de 2005.

Wikipedia. Carbon Dioxide, 2010 [en línea]. (Fecha de consulta 30 de Julio de 2010). Disponible en: http://en.wikipedia.org/wiki/ Carbondioxide

\section{Semblanza del autor}

José A. Raynal-Villaseñor. Es profesor titular del Departamento de Ingeniería Civil y Ambiental de la Universidad de las Américas, Puebla, ubicada en Cholula, Puebla. Obtuvo su doctorado en hidrología y recursos hidráulicos en la Universidad Estatal de Colorado en Estados Unidos de América. Es miembro del Sistema Nacional de Investigadores con Nivel I. Ha publicado varios libros y artículos arbitrados en revistas científicas periódicas y en congresos nacionales e internacionales. Es miembro de diversas asociaciones profesionales nacionales e internacionales, entre las que destacan la Academia Mexicana de Ciencias, la Academia de Ingeniería y la Academia de Ciencias de Nueva York. 